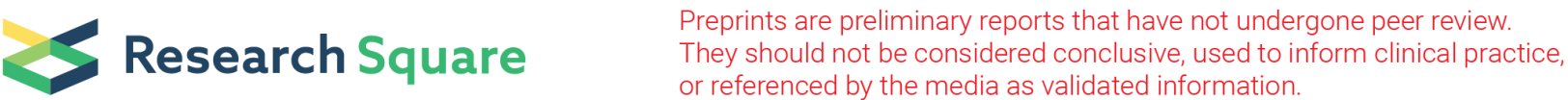

\section{Additive Effects of Evaluating Micro-Surface and Microvascular Patterns Using Magnifying Endoscopy with Narrow-Band Imaging for Gastric Cancer}

Yusuke Horiuchi ( $\nabla$ yusuke.horiuchi@jfcr.or.jp )

Cancer Institute Hospital

Toshiaki Hirasawa

Cancer Institute Hospital

Naoki Ishizuka

Cancer Institute Hospital

Junki Tokura

Cancer Institute Hospital

Mitsuaki Ishioka

Cancer Institute Hospital

Yoshitaka Tokai

Cancer Institute Hospital

Ken Namikawa

Cancer Institute Hospital

Shoichi Yoshimizu

Cancer Institute Hospital

Akiyoshi Ishiyama

Cancer Institute Hospital

Toshiyuki Yoshio

Cancer Institute Hospital

Junko Fujisaki

Cancer Institute Hospital

\section{Research Article}

Keywords: Diagnostic performance, Endoscopy, Endoscopic submucosal dissection, Gastric cancer

Posted Date: September 15th, 2021

DOI: https://doi.org/10.21203/rs.3.rs-882467/v1 
License: (c) (i) This work is licensed under a Creative Commons Attribution 4.0 International License. Read Full License 


\section{Abstract}

No studies have compared the performance of microvascular and micro-surface patterns alone with their combination in magnifying endoscopy with narrow-band imaging for diagnosing gastric cancer. This study aimed to clarify the difference in diagnostic performance between these methods. Thirty-three participating endoscopists underwent specialized training in magnifying endoscopy evaluated microvascular and micro-surface patterns for images of 106 cancerous and 106 non-cancerous cases. If classified as "irregular", the lesion was diagnosed as cancerous. To evaluate diagnostic performance, we compared the diagnostic accuracy, sensitivity, and specificity among the methods. Performance-related items did not significantly differ between the microvascular and micro-surface patterns. However, the diagnostic accuracy and sensitivity were significantly higher when using the combination of these methods than when using the microvascular pattern alone (percentage [95\% confidence interval]: $82.1 \%$ [76.4-86.7] vs. 76.4\% [70.3-81.6], $P=0.0005$; and $69.8 \%$ [60.5-77.8] vs. $63.2 \%$ [53.7-71.8], $P=0.0082$, respectively). The additive effects on diagnostic accuracy and sensitivity were $5.7 \%$ and $6.6 \%$, respectively. The combination of micro-surface and microvascular patterns has superior diagnostic accuracy and sensitivity for diagnosing gastric cancer than the evaluation method using microvascular pattern alone. Our results may contribute to improving the diagnosis of gastric cancers.

\section{Introduction}

Gastric cancer is among the most prevalent types of cancer and is associated with high mortality [1,2]. However, owing to recent advances in endoscopic technology, the number of gastric cancer cases detected at an early stage has increased and mortality rates have decreased [3-5]. Among these recent developments is magnifying endoscopy with narrow-band imaging (ME-NBI), which has superior diagnostic performance when combined with conventional endoscopy [6, 7]. Therefore, ME-NBI is currently the standard for diagnosing gastric cancer.

The vessel plus surface classification (VS classification) system used during ME-NBI has been reported to be useful for diagnosing gastric cancers $[7,8]$. In the VS classification system $[7,8]$, the demarcation line between the cancerous and non-cancerous areas is identified under low magnification, following which the target area is observed under high magnification, and both the microvascular pattern (MV) and micro-surface pattern (MS) are evaluated. Cancer is diagnosed if irregular findings are observed with respect to either the MV or MS. Meanwhile, some studies have reported that assessments based on MV alone are feasible for diagnosing gastric cancer [9-11]. At present, no previous study has compared the diagnostic performance between the combination of MS and MV and MV alone; therefore, this difference is not clear.

If there indeed exist additive effects of evaluating MS and MV, the results of this analysis can show the superiority of the combination of MS and MV over the evaluation method of using MV alone for diagnosing gastric cancer. In contrast, if high diagnostic performance can be obtained with MS or MV alone, the diagnostic system can be simplified by omitting the unnecessary component. In the present 
study, we aimed to evaluate the respective contributions of MS and MV for diagnosing patients with gastric cancer, as well as assess the difference between the combination of MS and MV and MV alone in diagnostic performance.

\section{Results}

A total of 106 lesions in 102 patients satisfied the inclusion and exclusion criteria (Fig. 1). Table 1 shows the background characteristics of the included patients. The median age was 71 years (interquartile range [IQR]: $61.8-77$, range: $26-87) ; 66$ (64.7\%) patients were male and $36(35.3 \%)$ were female. 
Table 1

Patient characteristics

\begin{tabular}{|c|c|c|c|}
\hline \multicolumn{4}{|c|}{102 cases, 106 lesions } \\
\hline Age & & & $71(61.8-77)[26-87]$ \\
\hline Sex (male/female) & & & 66 (64.7)/36 (35.3) \\
\hline \multirow[t]{4}{*}{ Location } & Upper third & & $26(24.5)$ \\
\hline & Middle third & & $59(55.7)$ \\
\hline & Lower third & & $17(16.0)$ \\
\hline & Gastric tube & & $4(3.8)$ \\
\hline \multirow[t]{4}{*}{ Macroscopic type } & Elevated & & $15(14.2)$ \\
\hline & Flat & & $5(4.7)$ \\
\hline & Depressed & & $83(78.3)$ \\
\hline & Complex & & $3(2.8)$ \\
\hline Tumor diameter & & & $14(9-20.3)(1.5-57)$ \\
\hline \multirow[t]{3}{*}{ Depth } & Intramucosal & & $90(84.9)$ \\
\hline & Submucosal & $<500 \mu \mathrm{m}$ & $12(11.3)$ \\
\hline & & $\geq 500 \mu \mathrm{m}$ & $4(3.8)$ \\
\hline \multirow[t]{2}{*}{ Ulcerative findings } & Present & & $5(4.7)$ \\
\hline & Absent & & $101(95.3)$ \\
\hline \multirow[t]{2}{*}{ Histological type } & Differentiated type & & $82(77.4)$ \\
\hline & $\begin{array}{l}\text { Undifferentiated } \\
\text { type }\end{array}$ & & $24(22.6)$ \\
\hline \multirow{4}{*}{$\begin{array}{l}\text { History of Helicobacter pylori } \\
\text { infection }\end{array}$} & Infected & & $97(91.5)$ \\
\hline & & $\begin{array}{l}\text { Non- } \\
\text { eradicated }\end{array}$ & $23(21.7)$ \\
\hline & & Eradiated & $74(76.3)$ \\
\hline & Uninfected & & $9(8.5)$ \\
\hline
\end{tabular}


The patients had undergone ME-NBI before endoscopic submucosal dissection (ESD), on a day different from the treatment day. Thirty-three endoscopists with specialized training analysed and provided a diagnosis (regular, irregular, absent, or inconclusive) for the MS and/or MV of each image. Table 2 shows the diagnostic agreement rates for all images, which were $84.8 \%$ for MV (IQR: 72.7-93.9) and $69.7 \%$ for MS (IQR: 51.5-87.9). The lower limit of the IQR for both MV and MS exceeded $50 \%$.

Table 2

Median diagnostic agreement rate for each finding in all images

\begin{tabular}{|ll|}
\hline MV & \\
\hline Total $(n=212)$ & $72.7 \%(60.6-81.8)[36.4-100]$ \\
\hline Cancerous area $(n=106)$ & $72.7 \%(57.6-87.9)[36.4-100]$ \\
\hline Non-cancerous area $(n=106)$ & $72.7 \%(60.6-81.8)[36.4-93.9]$ \\
\hline MS & $72.7 \%(54.5-81.8)[30.3-93.9]$ \\
\hline Total $(n=212)$ & $60.6 \%(51.5-78.8)[33.3-93.9]$ \\
\hline Cancerous area $(n=106)$ & $78.8 \%(63.6-84.8)[30.3-93.9]$ \\
\hline Non-cancerous area $(n=106)$ & Data are expressed as median (interquartile range) [range] \\
\hline MV microvascular pattern, MS micro-surface pattern \\
\hline
\end{tabular}

Table 3 shows the diagnoses based on MV and MS for the non-cancerous and cancerous images. Among the non-cancerous images, regular MS and MV findings were observed in 94 images (88.7\%). Among the cancerous images, both irregular MV and MS findings were observed in 49 images (46.2\%), irregular MV findings were observed only in 18 images (16.9\%), and irregular MS findings were observed only in 7 images (6.6\%). 
Table 3

$\mathrm{MV}$ and $\mathrm{MS}$ in each non-cancerous and cancerous image

\begin{tabular}{|llll|}
\hline MV & MS & Non-cancerous images $(\boldsymbol{n}=106)$ & Cancerous images $(\boldsymbol{n}=106)$ \\
\hline Regular & Regular & $94(88.7)$ & $29(27.4)$ \\
\hline Regular & Irregular & 0 & $5(4.7)$ \\
\hline Regular & Absent & 0 & $1(0.9)$ \\
\hline Regular & Inconclusive & 0 & 0 \\
\hline Irregular & Regular & 0 & $1(0.9)$ \\
\hline Irregular & Irregular & $6(5.7)$ & $49(46.2)$ \\
\hline Irregular & Absent & 0 & $17(16.0)$ \\
\hline Irregular & Inconclusive & 0 & 0 \\
\hline Absent & Regular & $1(0.9)$ & $1(0.9)$ \\
\hline Absent & Irregular & 0 & 0 \\
\hline Absent & Absent & 0 & 0 \\
\hline Absent & Inconclusive & 0 & 0 \\
\hline Inconclusive & Regular & $5(4.7)$ & 0 \\
\hline Inconclusive & Irregular & 0 & $2(1.9)$ \\
\hline Inconclusive & Absent & 0 & 0 \\
\hline Inconclusive & Inconclusive & 0 & $1(0.9)$ \\
\hline Data are presented as numbers $(\%)$ & \\
\hline MV microvascular pattern, MS micro-surface pattern & \\
\hline
\end{tabular}

Based on the results mentioned in Table 3, we calculated the diagnostic accuracy, sensitivity, specificity, positive predictive value (PPV), and negative predictive value (NPV). Subsequently, we compared the diagnostic performance between MV alone and MS alone and between MV alone and the combination of MS and MV (Table 4). For all items, there was no significant difference in the diagnostic performance between MV alone and MS alone. In contrast, diagnostic accuracy and sensitivity were significantly higher for the combination of MS and MV than for MV alone (accuracy: 82.1\% [95\% confidence interval [Cl]: 76.4-86.7] vs. $76.4 \%$ [95\% Cl: 70.3-81.6], $\mathrm{P}=0.0005$; sensitivity: $69.8 \%$ [95\% Cl: $60.5-77.8]$ vs. $63.2 \%$ [95\% Cl: 53.7-71.8], $\mathrm{P}=0.0082$, respectively). The additive effects on diagnostic accuracy and sensitivity were $5.7 \%$ and $6.6 \%$, respectively. 
Table 4

Comparison of diagnostic performance between MV alone and MS alone and between MV alone and the combination of MV and MS

\begin{tabular}{|c|c|c|c|c|c|}
\hline & 1. MV alone & 2. MS alone & 3. MV and MS & $p_{2)}^{p(1 \mathrm{vs} .}$ & $p_{3)}^{p(1 \mathrm{vs} .}$ \\
\hline $\begin{array}{l}\text { Accuracy, \% (95\% } \\
\mathrm{Cl})\end{array}$ & $\begin{array}{l}76.4(70.3- \\
81.6)\end{array}$ & $\begin{array}{l}73.6(67.3- \\
79.1)\end{array}$ & $\begin{array}{l}82.1(76.4- \\
86.7)\end{array}$ & 0.0273 & 0.0005 \\
\hline $\begin{array}{l}\text { Sensitivity, \% (95\% } \\
\mathrm{Cl})\end{array}$ & $\begin{array}{l}63.2(53.7- \\
71.8)\end{array}$ & $\begin{array}{l}52.8(43.4- \\
62.1)\end{array}$ & $\begin{array}{l}69.8(60.5- \\
77.8)\end{array}$ & 0.0278 & 0.0082 \\
\hline $\begin{array}{l}\text { Specificity, \% (95\% } \\
\mathrm{Cl})\end{array}$ & $\begin{array}{l}89.6(82.4- \\
94.1)\end{array}$ & $\begin{array}{l}94.3(88.2- \\
97.4)\end{array}$ & $\begin{array}{l}94.3(88.2- \\
97.4)\end{array}$ & 0.0253 & 0.0253 \\
\hline PPV, \% (95\% Cl) & $\begin{array}{l}91.8(83.2- \\
96.2)\end{array}$ & $\begin{array}{l}90.2(80.2- \\
95.4)\end{array}$ & $\begin{array}{l}92.4(84.4- \\
96.5)\end{array}$ & 0.7699 & $>0.9999$ \\
\hline NPV, \% (95\% Cl) & $\begin{array}{l}71.9(64.0- \\
78.7)\end{array}$ & $\begin{array}{l}66.2(58.4- \\
73.3)\end{array}$ & $\begin{array}{l}75.2(67.2- \\
81.8)\end{array}$ & 0.3116 & 0.5838 \\
\hline
\end{tabular}

In addition, as shown in Table 5, there were no significant differences in lesion characteristics between lesions correctly diagnosed using MV alone and lesions misdiagnosed using MV and correctly diagnosed using MS. 
Table 5

Comparison of lesion characteristics between cases correctly diagnosed using MV alone and those misdiagnosed using MV and correctly diagnosed using MS

\begin{tabular}{|c|c|c|c|c|}
\hline & & $\begin{array}{l}\text { Correctly } \\
\text { diagnosed using } \\
\text { MV alone }(n=67)\end{array}$ & $\begin{array}{l}\text { Misdiagnosed using MV } \\
\text { and correctly diagnosed } \\
\text { using MS }(n=7)\end{array}$ & $p$-value \\
\hline \multirow[t]{4}{*}{ Location } & Upper third & $7(10.5)$ & $2(28.6)$ & 0.4759 \\
\hline & Middle third & $40(59.7)$ & $3(42.9)$ & \\
\hline & Lower third & $16(23.9)$ & $2(28.6)$ & \\
\hline & Gastric tube & $4(6.0)$ & 0 & \\
\hline \multirow[t]{4}{*}{ Macroscopic type } & Elevated & $11(16.4)$ & $1(14.3)$ & 0.7951 \\
\hline & Flat & $5(7.5)$ & 0 & \\
\hline & Depressed & $48(71.6)$ & $6(85.7)$ & \\
\hline & Complex & $3(4.5)$ & 0 & \\
\hline \multirow[t]{2}{*}{ Tumour diameter } & $\geq 15 \mathrm{~mm}$ & $33(49.3)$ & $1(14.3)$ & 0.1158 \\
\hline & $<15 \mathrm{~mm}$ & $34(50.7)$ & $6(85.7)$ & \\
\hline \multirow[t]{2}{*}{ Depth } & Intramucosal & $57(85.1)$ & $6(85.7)$ & ${ }_{0.9999}$ \\
\hline & $\begin{array}{l}\text { Submucosal } \\
\text { invasion }\end{array}$ & $10(14.9)$ & $1(14.3)$ & \\
\hline \multirow{2}{*}{$\begin{array}{l}\text { Ulcerative } \\
\text { findings }\end{array}$} & Present & $3(4.5)$ & $2(28.6)$ & 0.0674 \\
\hline & Absent & $64(95.5)$ & $5(71.4)$ & \\
\hline \multirow[t]{2}{*}{ Histological type } & $\begin{array}{l}\text { Differentiated } \\
\text { type }\end{array}$ & $50(74.6)$ & $6(85.7)$ & $\overrightarrow{0} .9999$ \\
\hline & $\begin{array}{l}\text { Undifferentiated } \\
\text { type }\end{array}$ & $17(25.4)$ & $1(14.3)$ & \\
\hline \multirow{4}{*}{$\begin{array}{l}\text { History of } \\
\text { Helicobacter } \\
\text { pylori infection }\end{array}$} & Infected & & & 0.2389 \\
\hline & Non-eradicated & $15(22.4)$ & 0 & \\
\hline & Eradicated & $47(70.2)$ & $7(100)$ & \\
\hline & Uninfected & $5(7.5)$ & 0 & \\
\hline \multicolumn{5}{|c|}{ Data are presented as numbers (\%) } \\
\hline
\end{tabular}




\section{Discussion}

To the best of our knowledge, the present study is the first to evaluate the respective contributions of MS and MV for diagnosing gastric cancer, as well as the difference between the combination of MS and MV and $\mathrm{MV}$ alone in diagnostic performance.

Our findings indicate that there are cases that cannot be diagnosed without considering MS. Moreover, we observed no significant difference in the diagnostic performance between MV alone and MS alone. In other words, diagnoses determined based on MS may exhibit the same diagnostic performance as those made based on MV. While some previous reports have indicated that gastric cancer can be diagnosed based on MV alone $[9,10]$, none have investigated the usefulness of MS alone. The major strength of our study is that our findings highlight the importance of MS when diagnosing gastric cancer in clinical practice.

In addition, the diagnostic accuracy and sensitivity of the combination of MS and MV were significantly higher than those of MV alone, and the additive effects of evaluating MS and MV were present. The ability to identify cancerous lesions during endoscopic screening is paramount. Therefore, sensitivity is the most important factor contributing to the diagnostic performance. Our above-mentioned findings suggest that a diagnosis based on the combination of both patterns is useful for patients with gastric cancers in clinical practice. While the combination of MS and MV has been reported to be useful for diagnosing gastric cancers [7, 8], some studies have reported that assessments based on MV alone are feasible for diagnosis [9-11]. Furthermore, no previous reports have compared the diagnostic performance between the combination of MS and MV and MV alone. Given that our result shows the superiority of the combination of MS and MV over the evaluation method of using MV alone for diagnosing gastric cancer, our data support the application of the former in clinical practice.

Moreover, the lower limit of the IQR for the diagnostic agreement rate for both MV and MS exceeded $50 \%$. Namely, the responses aggregated in this study were considered the general answers of choice for the majority of endoscopists. Therefore, this result supports the reliability of the calculated diagnostic performance and the generalizability of our results and is one of the strengths of our study.

We also compared the lesion characteristics between lesions correctly diagnosed using MV alone and those misdiagnosed using MV and correctly diagnosed using MS to clarify whether the additive effects are associated with specific image attributes. However, we observed no significant differences in any items, which may have been owing to the small number of lesions correctly diagnosed based on the addition of MS to MV. While further studies are required, this result suggests that the diagnoses of gastric cancer should be made based on the combination of both patterns, regardless of lesion attributes.

This study had some limitations. First, the images were retrospectively collected from a single centre. Second, diagnoses were not made at the time of real-time endoscopy. Finally, as all patients underwent ESD, some lesions could have been overlooked. Meanwhile, the images used in this study were from consecutive cases wherein patients were examined at a cancer-specialized hospital and all images were 
evaluated by 33 endoscopists from 19 facilities nationwide. Therefore, the influence of bias was likely small, and the results of this study can be generalised. Furthermore, because the design of this study included a distinguishing feature between the cancerous area and the adjacent non-cancerous area, our findings indicated that it is possible to distinguish between adjacent non-cancerous gastric mucosa and gastric cancers, suggesting that our results can be useful for detecting gastric cancers. Therefore, despite the limitations of this study, we consider our results to be clinically meaningful at this stage. To address these limitations, we plan to prospectively evaluate the diagnostic performance (including an evaluation of the demarcation line) of MS alone, MV alone, and the combination of MS and MV during real-time endoscopy in collaboration with multiple centres. At such a time, the results of the present study will provide a valuable basis for comparison.

In conclusion, our findings demonstrated that the combination of MS and MV has superior diagnostic accuracy and sensitivity for diagnosing gastric cancer than the evaluation method using MV alone and that some cases cannot be diagnosed without considering MS. Our results support the application of the combination of MS and MV in clinical practice and may contribute to improving the diagnosis of gastric cancers.

\section{Methods}

This study was a post hoc analysis of results from our previous single-centre observational study [12]. We used images of 118 consecutive lesions in 114 patients who underwent ESD performed by a single endoscopist (Y.H.) from November 2016 to July 2019. Patient images and information were extracted from electronic medical records. ME-NBI was performed before treatment (on a day different from the treatment day). Before the examination, a soft hood (MB-46; Olympus Medical Systems) was mounted on the tip of the endoscope to enable the endoscopist to consistently fix the mucosa at approximately 2 $\mathrm{mm}$. We first performed endoscopy with white-light imaging, following which ME-NBI was performed to diagnose the cancerous and non-cancerous areas. In particular, the demarcation line between the cancerous and non-cancerous areas was identified under low magnification, following which the target area was observed under high magnification. Finally, endoscopy was performed following indigo carmine spraying.

The inclusion criterion was as follows: cases with ME-NBI images at the utmost oral side of the cancerous area and the adjacent non-cancerous area (one image of each cancerous and non-cancerous area per case). The exclusion criteria included cases without ME-NBI images and those with unclear images owing to the presence of mucus, blood, or halation.

According to the gastric cancer treatment guidelines [13], the cancerous and non-cancerous regions were confirmed in all cases based on post-ESD pathological results, which are considered the gold standard. All images were selected by one instructor of the Japan Gastroenterological Endoscopy Society (JGES) (Y.H.). In addition, another instructor of the JGES (T.H.) confirmed that all images met the inclusion criteria. GIF-H260Z and GIF-H290Z (Olympus Medical Systems, Tokyo, Japan) were used for ME-NBI. 


\section{Endoscopists involved in diagnosing the images and the diagnostic method}

Thirty-three endoscopists with specialized training in ME-NBI across 19 institutions participated in the diagnostic process. The VS classification system was used for diagnosis $[7,8]$. The VS classification system was established based on diagnoses made by endoscopists with ME-NBI training at specialized facilities [7]. A previous study has reported that diagnostic performance is better among these endoscopists than among those without training [14]. Therefore, because diagnoses by endoscopists without specialized training in ME-NBI may not adequately reflect the accuracy of the ME-NBI diagnosis, endoscopists with specialized training in ME-NBI were selected.

Each endoscopist evaluated each image using the terms regular, irregular, absent, or inconclusive. If either MV or MS was defined as "irregular," the lesion was diagnosed as cancerous; the lesion was not diagnosed as cancerous in the presence of other findings. Representative ME-NBI images in which the VS classification system was used are shown in Fig. 2.

In our previous study [12], the diagnostic results of cancer or non-cancer provided by each endoscopist were aggregated to calculate the diagnostic performance among all participating endoscopists. In the present study, the diagnostic results (regular, irregular, absent, or inconclusive) for the MS and/or MV of each image were collected using the original data of the previous study, and the diagnostic performance based on MS and/or MV was calculated for all images.

\section{Evaluation criteria}

This study was conducted in accordance with the Standards for the Reporting of Diagnostic Accuracy Studies 2015 guidelines [15]. For each image, the classification (regular, irregular, absent, or inconclusive) with the greatest frequency of response among the endoscopists was regarded as the final diagnosis. When multiple classifications exhibited the maximum number of answers, the final diagnosis was regarded as inconclusive. In contrast, if the agreement rate among the endoscopists for each image was low, the reliability of the diagnosis was deemed low and generalization was considered difficult.

Therefore, we also calculated the diagnostic agreement rate for each image, which was defined as the ratio of the maximum number of answers for a given classification to all answers.

We calculated the median and IQR of the diagnostic agreement rate for all images, which were confirmed based on MV and MS. MV and MS diagnoses were then aggregated for each of the cancer and noncancer images.

Based on the aggregated results, we calculated the diagnostic accuracy, sensitivity, specificity, PPV, and NPV for the diagnosis based on MV alone (irregular pattern indicative of cancer), MS alone (irregular pattern indicative of cancer), and the combination of MS and MV (cancer diagnosed if either had an irregular pattern). Subsequently, we compared the diagnostic performance between MV alone and MS alone and between MV alone and the combination of MS and MV. The classification of "inconclusive" was considered an incorrect answer. 
We defined accuracy as follows: (number of correctly diagnosed cancerous lesions among actual cancerous lesions plus number of correctly diagnosed non-cancerous tissues among actual noncancerous tissues)/total number of images.

In addition, to identify the factors that contributed to the additive effects in cancer images, we compared lesion characteristics (location, macroscopic type, tumour diameter, depth, ulcerative findings, histological type, history of Helicobacter pylori infection) between the cases that were correctly diagnosed using MV alone and those that were misdiagnosed using MV and correctly diagnosed using MS. The cutoff values for tumour diameter were determined with reference to the median value for all tumours.

H. pylori-uninfected (HPU) cases were defined as follows: 1) no prior H. pylori eradication, 2) negative results on the urea breath test (UBIT, Otsuka, Tokushima, Japan), 3) negative results for the H. pylori

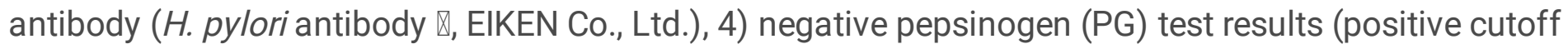
level: $\mathrm{PGI} \leq 70 \mathrm{ng} / \mathrm{mL}$ and $\mathrm{PGI} / \mathrm{II}$ ratio $\leq 3), 5$ ) endoscopically confirmed positive regular arrangement of collecting venules in the lower gastric body [16], and 6) histologically confirmed HPU and negative inflammatory cell infiltration activity based on the updated Sydney system [17]. Cases that did not meet these criteria were considered to have a history of $H$. pylori infection.

Inclusion criteria for the $H$. pylori-infected with eradication group were as follows: negative for $H$. pylori

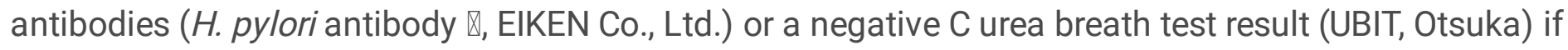
they underwent $H$. pylori eradication at our or another hospital; confirmed negative result for urea breath test performed at least 4 weeks after initiating $H$. pylori eradication if they were positive for $H$. pylori antibodies or had a positive urea breath test result at the first examination at our hospital. The H. pylori infection without eradication group did not meet these inclusion criteria.

\section{Ethical considerations}

This study was approved by the institutional review board of the Cancer Institute Hospital in Tokyo, Japan (approval no. 2019-1032) and was performed in accordance with the principles embodied in the Declaration of Helsinki and its later amendments. While recording the data for this study, all personal identifying information was removed. Informed consent was obtained from each patient for the use of pathological specimens and imaging data for research purposes.

\section{Statistical analysis}

The median and IQR were used when calculating the diagnostic agreement rate for all images. McNemar tests with $95 \%$ Cls were used to compare the diagnostic accuracy, sensitivity, and specificity among diagnoses made based on MV alone, MS alone, and the combination of MS and MV. Fisher's exact tests with 95\% Cls were used to compare PPV and NPV among the three diagnostic methods.

The statistical significance level was set at $P<0.05 / 2$ using the Bonferroni method for two pairwise comparisons in the same population (MV alone vs. MS alone, and MV alone vs. the combination of MS and MV). Fisher's exact test was used when comparing lesion characteristics between cases correctly 
diagnosed based on MV alone and those correctly diagnosed based on the combination of MS and MV, with the level of statistical significance set at $P<0.05$. JMP v13.2 (SAS ${ }^{\circledR}$ Institute, Cary, NC, USA) was used to perform the analyses.

\section{Declarations}

\section{Data Availability}

The data are not available for public access because of patient privacy concerns, but they are available from the corresponding author on reasonable request.

\section{Acknowledgments}

The authors thank the 33 endoscopists from the 19 participating institutions for evaluating the images.

\section{Author contributions}

Conception and design: Y.H. and T.H. Acquisition of data: Y.H., J.T., M.I., Y.T., K.N., and S.Y. Analysis and interpretation of the data: Y.H. and N.I. Drafting of the article: Y.H. Critical revision of the article for important intellectual content: Y.H., T.H., N.I. , J.T., M.I., Y.T., K.N., S.Y., A.I., T.Y., T.T., and J.F. Statistical analysis: Y.H. and N.I. Final approval of the article: Y.H., T.H., N.I., J.T., M.I., Y.T., K.N., S.Y., A.I., T.Y., and J.F. Study supervision: T.H. and J.F.

\section{Competing interests}

Yusuke Horiuchi have received research grants from Grant-in-Aid for Early-Career Scientists (21K15962) and personal fees for specific speaking and teaching commitments as honoraria from Olympus Corporation and Kaken Pharmaceutical Co., Ltd. Toshiyuki Yoshio has received research grants from the Takeda Science Foundation and a Grant-in-Aid for Scientific Research (C) (19K08408). Toshiaki Hirasawa, Naoki Ishizuka, Junki Tokura, Mitsuaki Ishioka, Yoshitaka Tokai, Ken Namikawa, Shoichi Yoshimizu, Akiyoshi Ishiyama, and Junko Fujisaki have no conflicts of interest or financial ties to disclose.

Funding: This study did not receive any funding from public, private, or non-for-profit organizations.

\section{References}

1. Torre, L. A. et al. Global cancer statistics. CA Cancer J. Clin, 65, 87-108 (2012).

2. Bray, F. et al. Global cancer statistics GLOBOCAN estimates of incidence and mortality worldwide for 36 cancers in 185 countries. CA Cancer J. Clin, 68, 394-424 (2018).

3. Ferro, A. et al. Worldwide trends in gastric cancer mortality (1980-2011), with predictions to 2015, and incidence by subtype. Eur J Cancer, 50, 1330-1344 (2014). 
4. Allum, W. H. et al. Guidelines for the management of oesophageal and gastric cancer., $60,1449-$ $1472(2011)$.

5. Thrumurthy, S. G., Chaudry, M. A., Hochhauser, D. \& Mughal, M. The diagnosis and management of gastric cancer. BMJ, 347, f6367 (2013).

6. Ezoe, Y. et al. Magnifying narrow-band imaging is more accurate than conventional white-light imaging in diagnosis of gastric mucosal cancer., 141, 2017-2025 (2011).

7. Yao, K., Anagnostopoulos, G. K. \& Ragunath, K. Magnifying endoscopy for diagnosing and delineating early gastric cancer. Endoscopy, 41, 462-467 (2009).

8. Muto, M. et al. Magnifying endoscopy simple diagnostic algorithm for early gastric cancer (MESDAG). Dig. Endosc, 28, 379-393 (2016).

9. Nakayoshi, T. et al. Magnifying endoscopy combined with narrow band imaging system for early gastric cancer: correlation of vascular pattern with histopathology (including video). Endoscopy, 12, 1080-1084 (2004).

10. Yokoyama, A. et al. Novel narrow-band imaging magnifying endoscopic classification for early gastric cancer.Dig. Liver Dis.42,704-708.

11. Horiuchi, Y. et al. Additive effect of magnifying endoscopy with narrow-band imaging for diagnosing mixed-type early gastric cancers. Dig. Dis. Sci, 65, 591-599 (2020).

12. Horiuchi, Y. et al. Diagnostic performance in gastric cancer is higher using endocytoscopy with narrow-band imaging than using magnifying endoscopy with narrow-band imaging., 24, 417-427 (2021).

13. . Japanese Gastric Cancer Association. Japanese gastric cancer treatment guidelines 2018 (5th edition). Gastric Cancer 24, 1-21 (2021).

14. Nakanishi, H. et al. Evaluation of an e-learning system for diagnosis of gastric lesions using magnifying narrow-band imaging: a multicenter randomized controlled study. Endoscopy, 49, 957967 (2017).

15. Cohen, J. F. et al. STARD 2015 guidelines for reporting diagnostic accuracy studies: explanation and elaboration. BMJ Open, 6, e012799 (2016).

16. Yagi, K., Nakamura, A. \& Sekine, A. Characteristic endoscopic and magnified endoscopic findings in the normal stomach without Helicobacter pylori infection. J. Gastroenterol. Hepatol, 17, 39-45 (2002).

17. Dixon, M. F., Genta, R. M., Yardley, J. H. \& Correa, P. The updated Sydney System. International Workshop on the Histopathology of Gastritis, Houston 1994. Am. J. Surg. Pathol. 20,11611181(1996).

\section{Figures}


Cases in which endoscopic submucosal dissection was performed

110 cases, 114 lesions

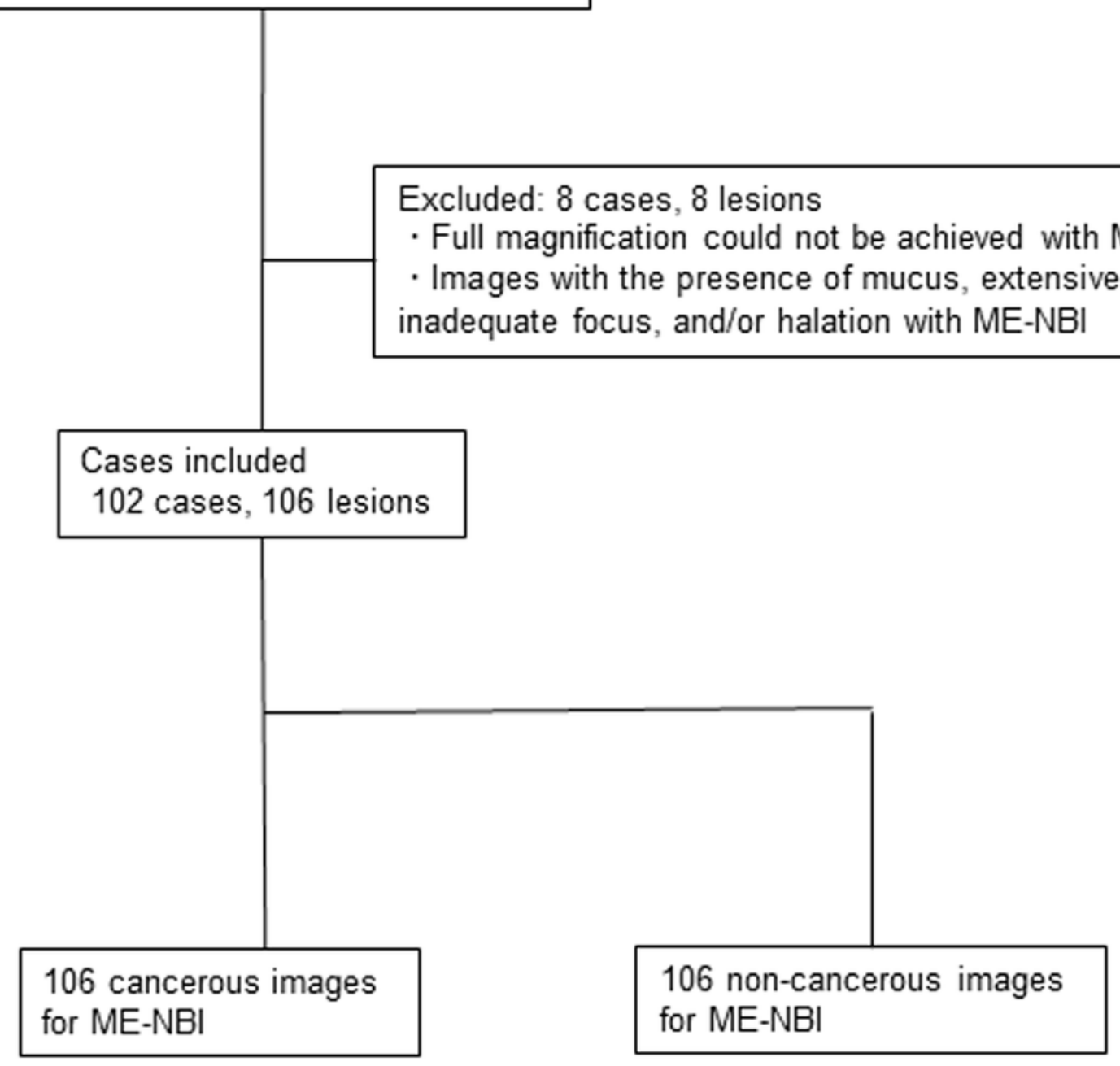

Fig.1

Figure 1

Patient flow diagram 

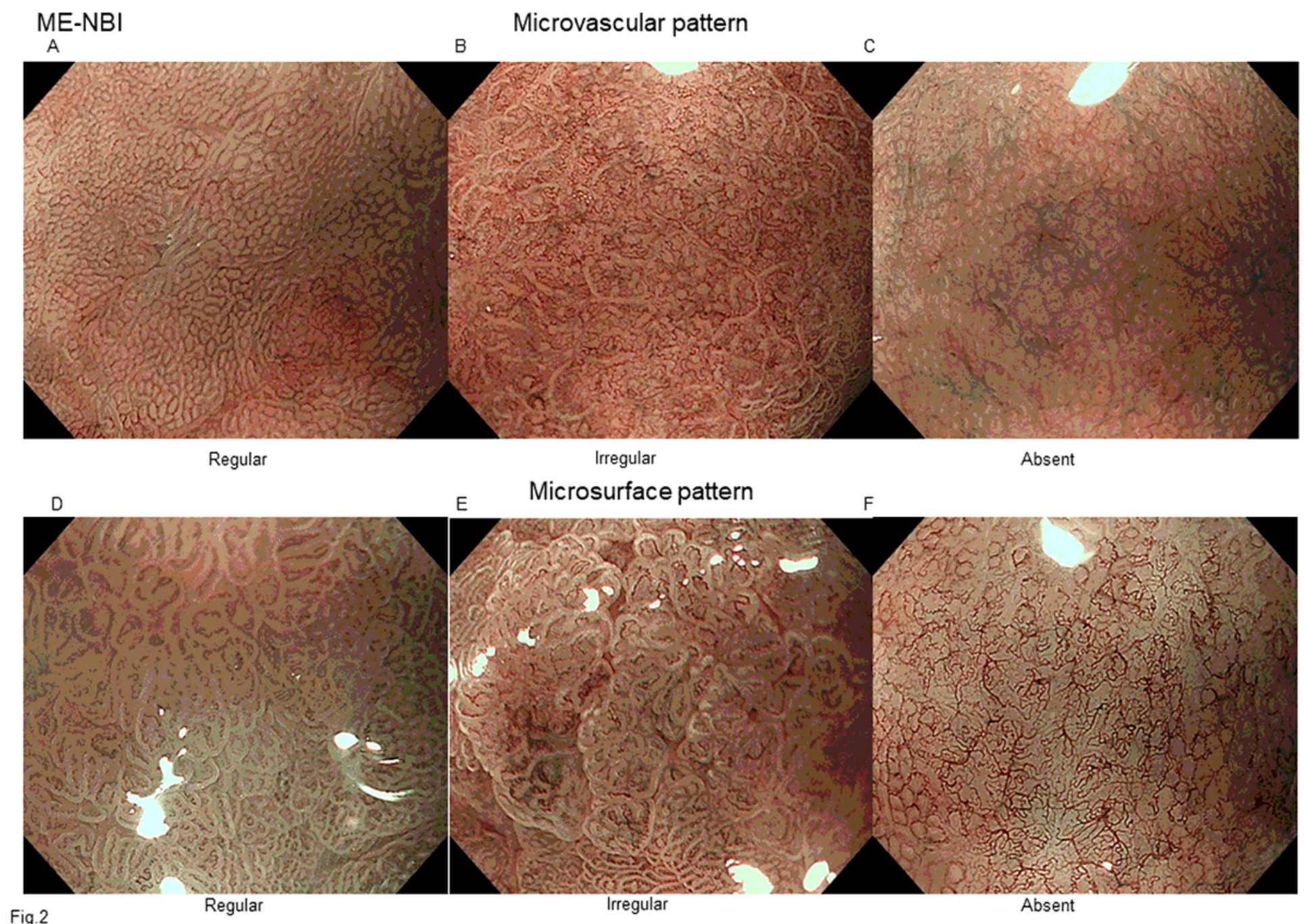

Fig. 2

$$
\text { Regular }
$$

Irregular

Absent

\section{Figure 2}

Microvascular pattern and micro-surface pattern classification (VS classification). The VS classification system is used to distinguish between cancerous lesions and non-cancerous tissues in patients undergoing magnifying endoscopy with narrow-band imaging. If either the microvascular pattern $(\mathrm{V})$ or the micro-surface pattern (S) is classified as "irregular", the lesion is diagnosed as cancerous. The details of the classification are as follows. Microvascular pattern: a uniform blood vessels (regular); b blood vessels that expand locally and are non-uniform (irregular); and c no vascular findings (absent). Microsurface pattern: $d$ uniform surface structure (regular); e non-uniform surface structure of different sizes (irregular); and $f$ no surface structure (absent) 